\title{
MiR-330-3p functions as a tumor suppressor that regulates glioma cell proliferation and migration by targeting CELF1
}

\author{
Hongbin Wang ${ }^{1}$, Guijing $\mathrm{Li}^{2}$, Tao $\mathrm{Li}^{1}$, Naizhu Wang ${ }^{1}$, Jingkun Wu ${ }^{1}$, Hua Zhi ${ }^{2}$
}

\author{
1Department of Neurosurgery, Affiliated Hospital of Hebei University of Engineering, \\ Handan, Hebei, China \\ 2Department of Cardiology, Affiliated Hospital of Hebei University of Engineering, \\ Handan, Hebei, China
}

Submitted: 5 September 2017

Accepted: 24 December 2017

Arch Med Sci 2020; 16 (5): 1166-1175

DOI: https://doi.org/10.5114/aoms.2020.95027

Copyright (c) 2020 Termedia \& Banach

\section{Abstract}

Introduction: Glioma is a common type of neoplasm that occurs in the central nervous system. miRNAs have been demonstrated to act as critical regulators of carcinogenesis and tumor progression in multiple cancers, but the molecular mechanism of miR-330-3p in glioma remained unclear. The purpose of the study was to explore the role of miR-330-3p in glioma cell reproduction and migration.

Material and methods: The expression levels of miR-330-3p and CELF1 in 27 glioma tissue specimens and human glioma cell lines were examined by qRT-PCR and western blot. The TargetScan database was used to predict the relationship between miR-330-3p and CELF1. Then the target relationship was verified using dual-luciferase reporter assay. The effects of miR-330-3p/ CELF1 on glioma cell proliferation were evaluated by MTT and colony formation assay. Wound healing assay was employed to measure the migration ability of glioma cells.

Results: MiR-330-3p was found lowly expressed in glioma tissues and cells compared with adjacent tissues and normal astrocytes, while CELF1 expression was relatively high in the glioma tissues and cells. Dual-luciferase reporter assay confirmed that miR-330-3p could directly target CELF1. Furthermore, miR-330-3p could down-regulate the expression of CELF1, therefore suppressing glioma cell reproduction and migration.

Conclusions: MiR-330-3p inhibited the propagation and migration of glioma cells by repressing CELF1 expression.

Key words: glioma, miR-330-3p, CELF1, proliferation, migration.

\section{Introduction}

Glioma is a kind of common neoplasm in the central nervous system, with a high morbidity and mortality rate worldwide [1-3]. Currently, the combined treatment of tumor resection with radiation and chemotherapy has been considered as a primary therapeutic method and widely applied to the treatment of neoplasms. Despite tremendous progress in the treatment of glioma, the average 5-year survival rate remains at a low level among glioma patients [4-6].

MicroRNAs (miRNAs) are highly conserved, non-coding small RNA molecules composed of approximately 20 nucleotides, which are involved

\author{
Corresponding author: \\ Hua Zhi \\ Department of Cardiology \\ Affiliated Hospital of Hebei \\ University of Engineering \\ 81 Congtai Road \\ Handan 056029 \\ Hebei, China \\ Phone/fax: \\ +86 0310-8572318 \\ E-mail: ynsyjkk@126.com
}


in the process of tumor initiation and progression [7-9]. Numerous previous studies have found a variety of miRNAs aberrantly expressed in glioma tissues and cells, contributing to tumorigenesis or carcinogenesis [10-13]. miRNAs have been found to function as tumor suppressors or facilitators through binding to $3^{\prime} U T R s$ of target genes in multiple diseases [14-17]. They regulate glioma cell proliferation and metastasis by regulating corresponding target genes $[18,19]$. For instance, miR330-3p functions in different types of cancers and has been extensively studied [20, 21]. Mesci et al. reported that miR-330-3p enhances breast cancer cell invasion and metastasis abilities by targeting CCBE1 [20]. Meng et al. verified that miR-330-3p acted as an oncogenic mRNA in esophageal squamous cell carcinoma by repressing PDCD4 expression [22]. However, the function and the specific molecular mechanism of miR-330-3p in malignant gliomagenesis remain unknown, and to understand the effects of miR-330-3p on the biological functions of glioma cells is critical for the development of novel therapeutic strategies of glioma.

CELF1, also known as CUGBP1, is an RNA binding protein that belongs to the CUGBP embryonic lethal abnormal vision-like family. CELF1 can control the post-transcriptional regulation of genes involved in cell survival [23]. Recent studies have shown that CELF1 was abnormally expressed in malignant $T$ cells and laryngeal cancer cells [24, 25]. Its aberrant expression may suggest its possible oncogenic role in various cancers; for instance, Xia et al. found that overexpression of CELF1 promoted glioma cell growth [26]. In this study, we also found the overexpression of CELF1 in glioma cells, which facilitated glioma cell propagation and migration. The specific mechanism of action underlying how CELF1 affects gliomagenesis needs to be determined.

Based on the findings of previous studies, we attempted to explore the role of miR-330-3p and CELF1 in glioma. We hypothesized that CELF1 might be a potential target of miR-330-3p and miR-330-3p might influence glioma cell reproduction and migration via regulation of CELF1.

\section{Material and methods}

\section{Tissue specimens}

Twenty-seven pairs of glioma tissues and adjacent normal tissues were obtained from patients with glioma undergoing surgical resection in the Affiliated Hospital of Hebei University of Engineering between August 2014 and August 2016 (Table I). All glioma tissue specimens were confirmed based on histopathological evaluation. We froze all tissues in liquid nitrogen and conserved them at $-80^{\circ} \mathrm{C}$ for the following assay. The study obtained permission from the Research Ethics Committee of the Affiliated Hospital of Hebei University of Engineering and informed consent from all participants.

\section{Cell culture, transfection and grouping}

Glioma cell lines (U87, U251, and C6) and the normal astrocyte cell line HA (ATCC, Manassas, VA) were cultured in DMEM medium (GIBCO, Gaithersburg, MD) plus $10 \% \mathrm{FBS}, 100 \mathrm{U} / \mathrm{ml}$ penicillin, and $100 \mathrm{ng} / \mathrm{ml}$ streptomycin, followed by incubation at $37^{\circ} \mathrm{C}$. MiR-330-3p mimics, negative control (NC) and CELF1-CDNA were purchased from Shanghai GenePharma Ltd. (Shanghai, China). Briefly, $1 \times 10^{5}$ U251 cells were inoculated into a 6-well plate and cultured at $37^{\circ} \mathrm{C}, 5 \% \mathrm{CO}_{2}$ overnight, followed by transfection with miR-330-3p mimics and CELF1 cDNA using Lipofectamine 2000 reagents (Invitrogen). The grouping of cells in the experiment was as follows: (1) the control group

Table I. Association between miR-330-3p expression and clinical characteristics

\begin{tabular}{|c|c|c|c|c|}
\hline \multirow[t]{2}{*}{$\begin{array}{l}\text { Clinical } \\
\text { characteristics }\end{array}$} & \multirow[t]{2}{*}{$N$} & \multicolumn{2}{|c|}{$\begin{array}{l}\text { miR-330-3p } \\
\text { expression }\end{array}$} & \multirow[t]{2}{*}{$P$-value } \\
\hline & & Low & High & \\
\hline \multicolumn{5}{|l|}{ Sex: } \\
\hline Male & 14 & 9 & 5 & 0.8826 \\
\hline Female & 13 & 8 & 5 & \\
\hline Age (years): & & & & 0.8068 \\
\hline$\leq 60$ & 17 & 11 & 6 & \\
\hline$>60$ & 10 & 6 & 4 & \\
\hline Tumor location: & & & & 0.7781 \\
\hline Parenchyma & 17 & 11 & 6 & \\
\hline Ventricle & 10 & 7 & 3 & \\
\hline Tumor size: & & & & 0.8826 \\
\hline$\leq 3 \mathrm{~cm}$ & 14 & 9 & 5 & \\
\hline$>3 \mathrm{~cm}$ & 13 & 8 & 5 & \\
\hline WHO grade*: & & & & 0.0341 \\
\hline$I+I I$ & 13 & 5 & 8 & \\
\hline III + IV & 14 & 11 & 3 & \\
\hline Karnofsky score*: & & & & $<0.0001$ \\
\hline$\leq 80$ & 16 & 15 & 1 & \\
\hline$>80$ & 11 & 2 & 9 & \\
\hline Resection range: & & & & 0.7215 \\
\hline Total resection & 15 & 9 & 6 & \\
\hline Local resection & 12 & 8 & 4 & \\
\hline
\end{tabular}

${ }^{*} p<0.05$, performed by $\chi^{2}$ test. 
(without transfection); (2) the NC group (transfection with antisense sequence); (3) the miR-330$3 p$ group (transfection with miR-330-3p mimics); (4) CELF1 group (transfection with CELF1 CDNA) and (5) miR-330-3p + CELF1 group (co-transfection with miR-330-3p mimics and CELF1 cDNA).

\section{qRT-PCR}

Trizol reagent and NanoDrop 1000 (Thermo Fisher Scientific) were respectively used for the extraction and quantification of total RNA. Total RNA was subjected to reverse transcription by using the PrimeScript RT reagent kit (Takara, Japan). Expression levels of miR-330-3p were measured using a TaqMan microRNA assay (Applied Biosystems, San Diego, USA). U6 (RUN6B) was assessed as an endogenous control. CELF1 mRNA levels were quantified by a SYBR Green PCR kit (TaKaRa, Dalian, China). GAPDH was used as an endogenous control to normalize the amount of total mRNA in each sample. The primer sequences used were as follows: for CELF1 5'-TCCTGCCGTTTGTTCATCGTT-3' (forward) and 5'-TTTCCCCTTCAGCAGTCGTTC-3' (reverse); for GAPDH: 5'-TATTGTTGCCATCAATGACCC-3' (forward) and 5'-ACTCCACGACGTACTCAGC-3' (reverse). MiR330-3p and CELF1 expression was quantified using the $2^{-\Delta \Delta C t}$ method.

\section{Western blot}

SDS-PAGE and bicinchoninic acid (BCA) Kit (Sigma-Aldrich, St. Louis, Missouri, USA) were used to separate cell lysate and quantify protein concentration. Proteins were transferred from SDS-PAGE gel to PVDF membranes, which were then soaked in $5 \%$ nonfat milk and incubated with antibodies against CELF1 and GAPDH (CELF1, GAPDH, $1: 1000,1: 2000$, Abcam, Cambridge, UK) at $4^{\circ} \mathrm{C}$ for $24 \mathrm{~h}$. Afterwards, the membranes were rinsed with TBST three times and incubated with HRPlabeled mouse anti-rabbit secondary antibody (1 : 2000) for $1.5 \mathrm{~h}$ at $37^{\circ} \mathrm{C}$. After washing with TBST for $15 \mathrm{~min}$, the immunoreactive proteins were visualized and analyzed by enhanced chemiluminescence $(\mathrm{ECL})$ western blotting detection reagent (GE Healthcare, Milwaukee, WI, USA).

\section{Dual-luciferase reporter gene assay}

To validate the relationship between miR-330 $3 p$ and CELF1, we first predicted the binding site of hsa-miR-330-3p on 3'UTR of CELF1 by means of the TargetScan database (http://www.targetscan.Org/). Meanwhile, dual-luciferase reporter gene assay was also used to further validate the correlation between miR-330-3p and CELF1. The wild type (wt) or mutated (mut) 3'-UTR of CELF1 and the predicted binding site of miR-330-3p were amplified and cloned into the pmirGLO vectors (Thermo Fisher Scientific). Then, we co-transfected pmirGLO recombinant vector and miR-330-3p mimics or negative control mimics into glioma cells and the relative Luciferase activity was detected after 48-hour transfection.

\section{MTT assay}

The cell proliferation assay was performed with an MTT kit (Sigma, St. Louis, Mo, USA). We first inoculated glioma cells with different transfection into a 96-well plate $\left(1 \times 10^{3}\right.$ cells/well $)$ Subsequently $20 \mu \mathrm{l}$ of MTT (15 mg/ml Sigma) was added to each well and kept for $3 \mathrm{~h}$. After the supernatants were discarded, $150 \mu \mathrm{l}$ of dimethyl sulfoxide (Sigma, St. Louis, Mo, USA) was added to each well. Lastly, the absorbance value of each well was assessed at $490 \mathrm{~nm}$. The assay was carried out three times.

\section{Colony formation assay}

Glioma cells were put into $60 \mathrm{~mm}$ culture dishes and incubation was performed in a standard environment for 7 days. Each group had six reduplicative wells. $4 \%$ paraformaldehyde and $0.1 \%$ crystal violet were added to the cells for fixation and staining. The colonies were observed through a microscope. All reactions were conducted three times.

\section{Wound healing assay}

We seeded glioma cells into 6-well plates $\left(4 \times 10^{5}\right.$ cells/well) $24 \mathrm{~h}$ post-transfection. Following adhesion, the medium was removed and the monolayer of cells was scraped with a $1000 \mu \mathrm{l}$ pipette tip. After rinsing with PBS twice and incubation for $24 \mathrm{~h}$, the scratch healing area was photographed by an inverted microscope and cell migration rates were measured at $0 \mathrm{~h}$ and $24 \mathrm{~h}$ respectively.

\section{Xenograft tumor assay}

Ten immunodeficient female nude mice (1520 g, Beijing Laboratory Animal Center, Beijing, China) were used to test the effects of miR-330$3 p$ on glioma in vivo. These nude mice were randomly divided into two groups (5 mice per group). Approximately $2 \times 10^{6}$ logarithmically growing glioma cells stably expressing NC or miR-330-3p mimics were subcutaneously injected into the nude mice. After 3 weeks, the subcutaneous tumors were stripped and weighed. Tumor volume was calculated according to the formula: $V\left(\mathrm{~mm}^{3}\right)$ $=\left(a \times b^{2}\right) / 2$ (a represents the longest axis and $b$ the shortest axis). The ethics committee of the Animal Experimental Center of Affiliated Hospital of Hebei University approved this study. 


\section{Statistical analysis}

Statistical analysis was conducted using SPSS 21.0 software (IBM, Chicago, Illinois, USA). All results were displayed as mean \pm standard deviation (SD). Normally distributed measurement data were analyzed by Student's $t$-test or one-way ANOVA, otherwise by the Mann-Whitney rank sum test. $P$-value $<0.01$ or 0.05 denoted statistical significance.

A

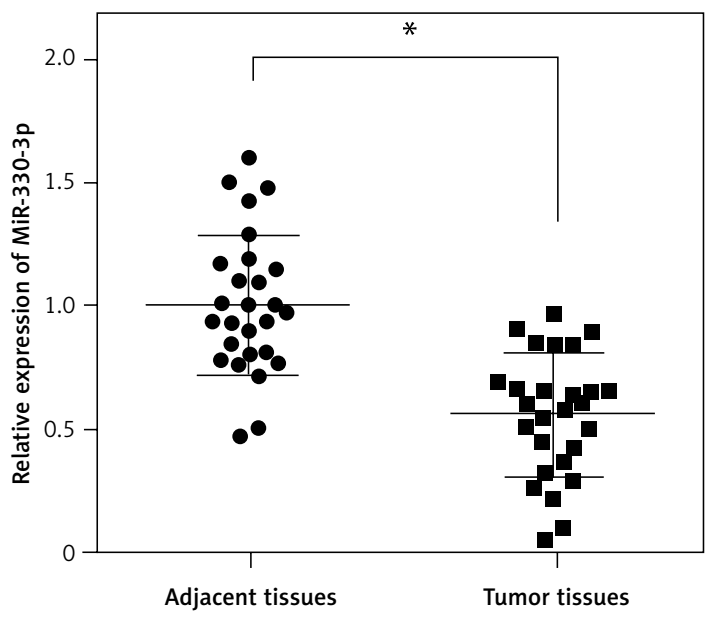

C

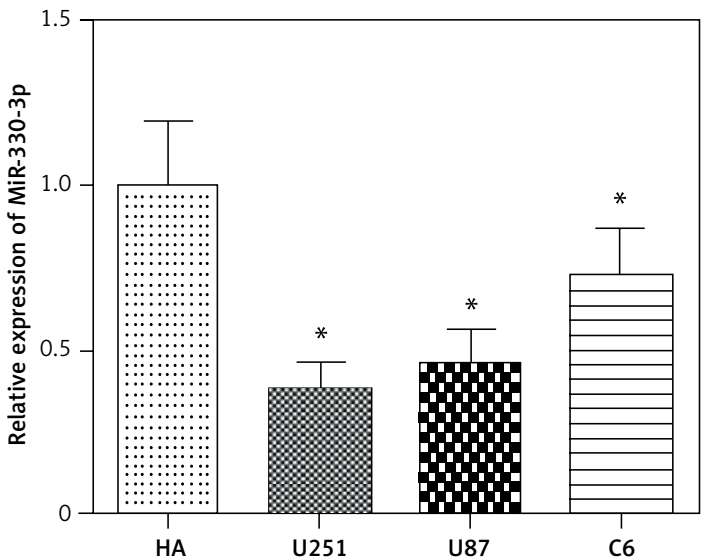

\section{Results}

MiR-330-3p was lowly expressed in glioma tissues and cells, while CELF1 was highly expressed in glioma tissues and cell lines.

We analyzed miR-330-3p and CELF1 expressions in tissues specimens using GRT-PCR and western blotting assay. As shown in Figures $1 \mathrm{~A}, \mathrm{~B}$, glioma tissues presented a significantly lower expression

B
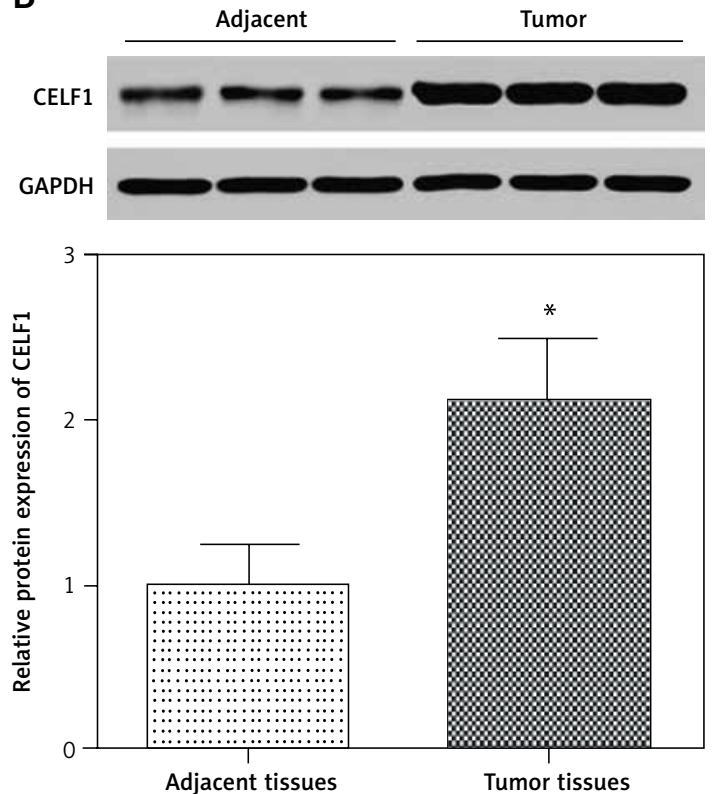

D

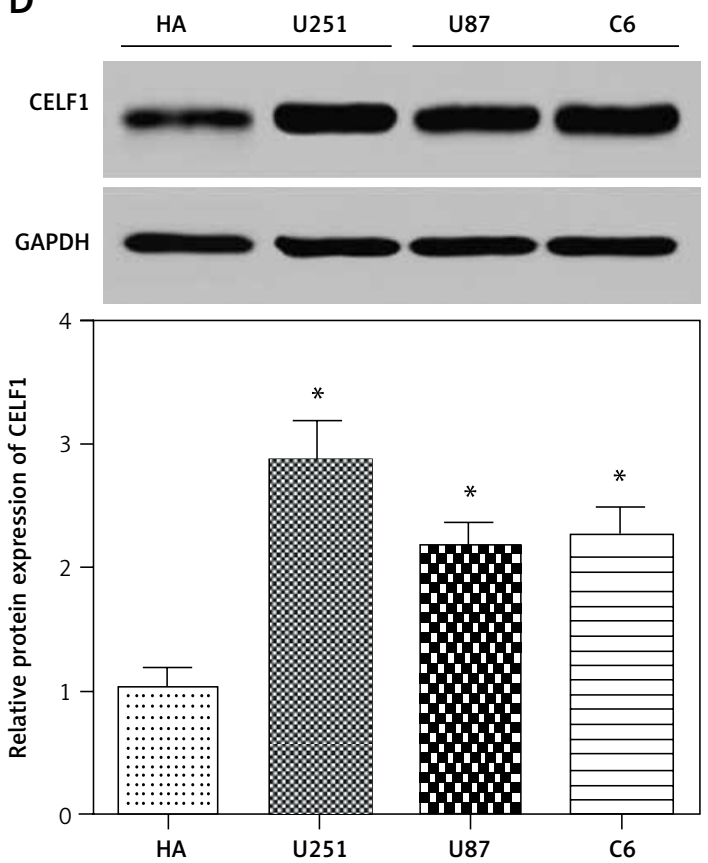

Figure 1. MiR-330-3p was lowly expressed in glioma tissues and cell lines, while CELF1 was highly expressed in glioma tissues and cell lines. A - The expression level of miR-330-3p mRNA in glioma tissues was significantly lower than that in adjacent normal tissues detected by GRT-PCR. B - The expression level of CELF1 protein was remarkably higher in glioma tissues in comparison with that of normal tissues detected by western blot. $\mathbf{C}-$ The expression level of miR-330-3p mRNA was found to be significantly lower in glioma cell lines (U87, U251 and C6) compared with that of normal cell line (HA cells) using qRT-PCR. D - The protein expression level of CELF1 was higher in glioma cell lines than in normal cell line detected by western blot. ${ }^{\star} P<0.05$, compared with adjacent tissues and normal cell line (HA) 
level of miR-330-3p compared with normal tissues $\left({ }^{*} p<0.05\right)$, whereas CELF1 expression was remarkably higher in in glioma tissues $\left({ }^{*} p<0.05\right)$. Likewise, the expression levels of miR-330-3p in glioma cell lines (U87, U251 and C6) were significantly lower compared with that in HA cells $\left({ }^{*} p<0.05\right)$ (Figure $1 \mathrm{C}$ ). Moreover, results from western blot indicated that CELF1 protein expression in glioma cell lines was remarkably higher in comparison with normal cells, especially in the U251 cell line ( ${ }^{*} p<0.05$, Figure $\left.1 \mathrm{D}\right)$. Overall, compared with U87 and C6, U251 had higher expression of miR-330-3p and lower expression of CELF1, which made it a proper subject to adapt the change of miR-330-3p and CELF1 in our further study. Therefore, the U251 cell line was chosen for the subsequent experiments.

\section{MiR-330-3p directly modulated CELF1 by targeting its 3'UTR}

We performed prediction analysis for binding sites of miR-330-3p on 3'UTR CELF1 using TargetScan. As shown in Figure $2 \mathrm{~A}$, CELF1 was one of the potential targets of miR-330-3p. Meanwhile, results of dual-luciferase reporter gene assay indicated that the luciferase activities of U251 cells transfected with CELF1 3'UTR-WT vectors were significantly weaker in the miR-330-3p mimic group than in the NC group. A difference of luciferase activities of $U 251$ cells transfected with CELF1 3'UTR-MUT vectors was not found between the NC group and the miR-330-3p group (Figure 2 B).

\section{MiR-330-3p suppressed glioma cell growth and migration by targeting CELF1}

To evaluate the functions of miR-330-3p and CELF1 in glioma cell growth and migration, we

A

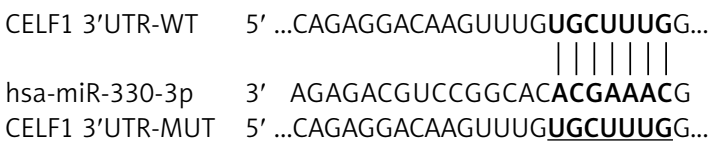

examined the expression of miR-330-3p in cells. Results from qRT-PCR revealed that the expression of miR-330-3p mRNA in the miR-330-3p group and miR-330-3p + CELF1 group was significantly up-regulated in comparison with the control group after transfection $\left({ }^{*} p<0.05\right.$, Figure $3 \mathrm{~A}$ ). Furthermore, western blot showed that CELF1 protein expression significantly decreased in the miR-330-3p mimic group, while CELF1 expression in cells transfected with CELF1 CDNA dramatically increased. MiR-330-3p could down-regulate the expression level of CELF1 through targeting CELF1 $\left({ }^{*} p<0.05\right.$, Figure $\left.3 \mathrm{~B}\right)$. In addition, according to the results of MTT assay, overexpression of miR-330$3 p$ significantly inhibited glioma cell viability or growth, while overexpression of CELF1 significantly promoted glioma cell viability or growth in comparison with the control group $\left({ }^{*} p<0.05\right.$, Figure $\left.3 C\right)$. Similarly, the colony formation assay demonstrated that miR-330-3p overexpression significantly repressed the proliferation ability of glioma cells, while CELF1 overexpression facilitated glioma cell proliferation. MiR-330-3p could suppress glioma cell proliferation via downregulation of CELF $\left({ }^{*} p<0.05\right.$, Figure $\left.3 \mathrm{D}\right)$. Meanwhile, the wound healing assay indicated that the migration rate of cells in the miR-330-3p overexpression group was significantly lower compared with the NC group, suggesting that miR-330-3p could suppress glioma cancer cell migration. On the other hand, the wound healing area in the CELF1 group was re markably larger in comparison with the control group, which indicated that CELF1 could promote glioma cancer cell migration $\left({ }^{*} p<0.05\right.$, Figure $3 \mathrm{E}$ ). After U251 cells were co-transfected with miR330-3p and CELF1, no significant difference of cell migration was found between the miR-330-3p +

B

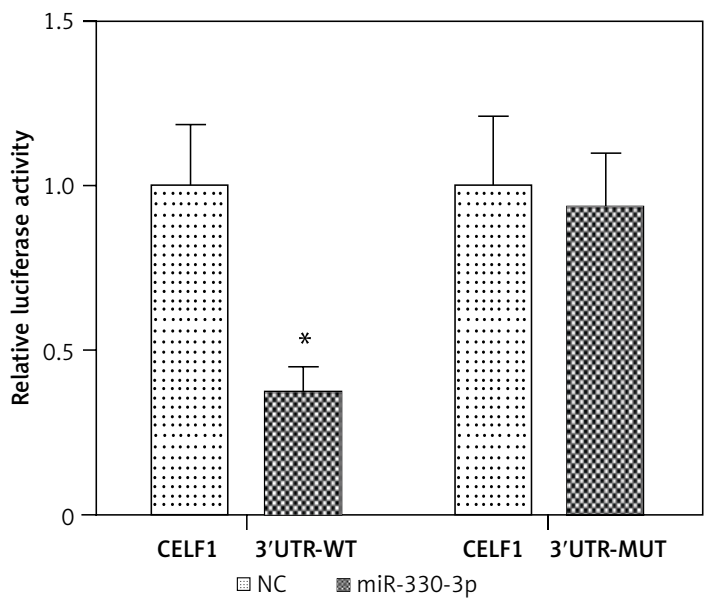

Figure 2. MiR-330-3p directly modulated CELF1 by targeting its 3'UTR. A - Alignment of the miR-330-3p putative binding site and corresponding mutated site on CELF1 3'UTR. B - The luciferase activity of U251 cells transfected with CELF1 3'UTR-WT vectors was significantly weaker in the miR-330-3p mimic group than in the NC group. Difference of luciferase activities of U251 cells transfected with CELF1 3'UTR-MUT vectors was not found between NC group and miR-330-3p group. ${ }^{*} P<0.05$, compared with NC group 
A

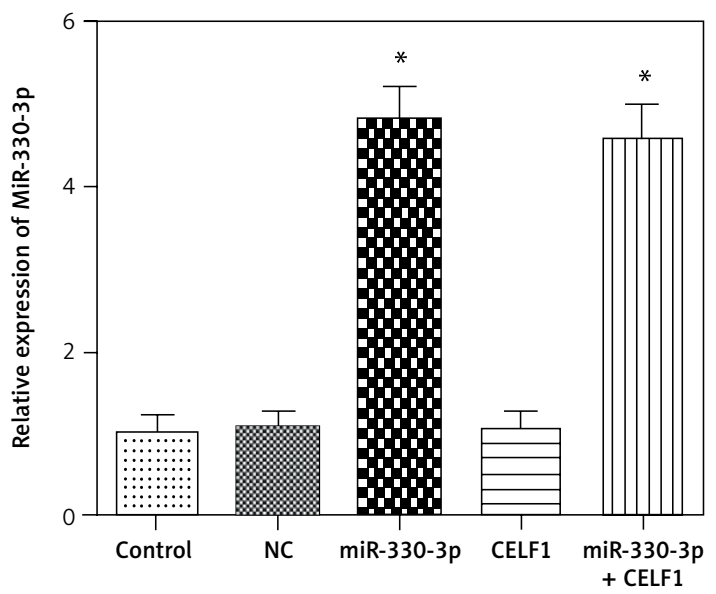

C

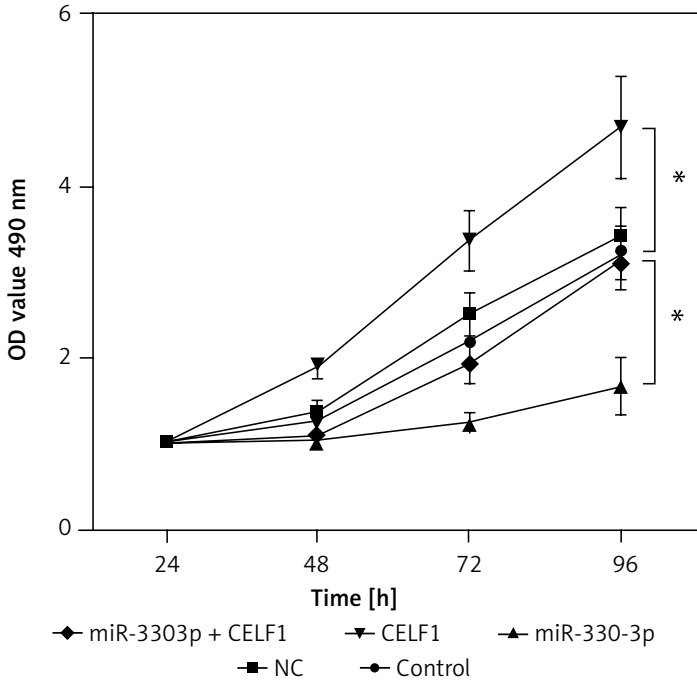

Figure 3. MiR-330-3p suppressed glioma cell growth and migration by targeting CELF1. A - The expression of miR-330-3p in miR-330-3p group and miR-330-3p + CELF1 group was significantly upregulated in comparison with control group after transfection detected by qRT-PCR. B - Western blot displayed that the expression level of CELF1 protein significantly decreased in cells transfected with miR-330-3p mimics, while CELF1 expression in cells transfected with CELF1 CDNA dramatically increased. The expression level of CELF1 protein in the miR-330-3p + CELF1 group was no different from the control group. C - MTT assay indicated that overexpression of miR-330-3p inhibited glioma cell viability or growth, while overexpression of CELF1 significantly promoted glioma cell viability or growth. MiR-330-3p exerted an inhibitory influence on glioma cell viability by downregulating CELF. D - Colony formation assay revealed that overexpression of miR-330-3p significantly repressed the proliferation ability of glioma cells, while CELF1 overexpression facilitated glioma cell proliferation. MiR-330-3p could suppress glioma cell proliferation via downregulation of CELF. ${ }^{*} P<0.05$, compared with the control group
B
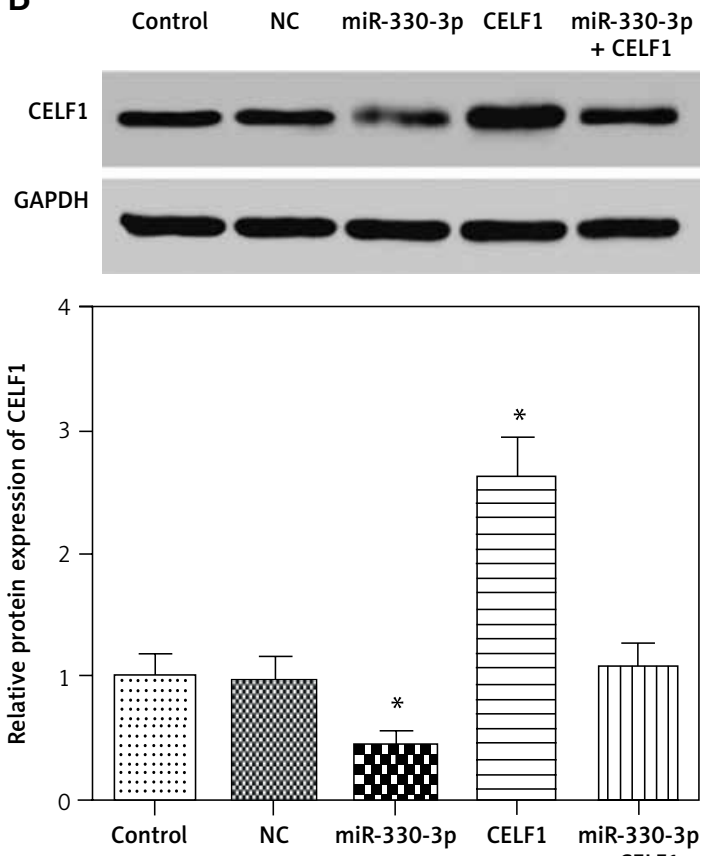

D

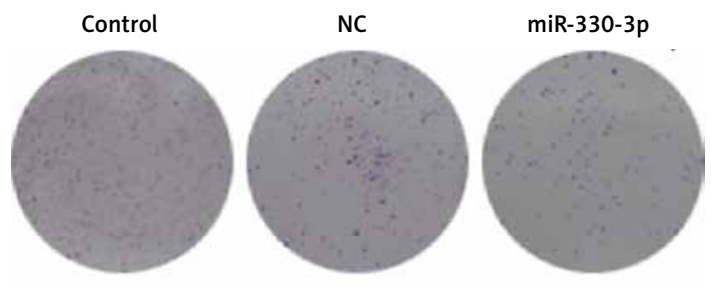

CELF1 miR-330-3p + CELF1
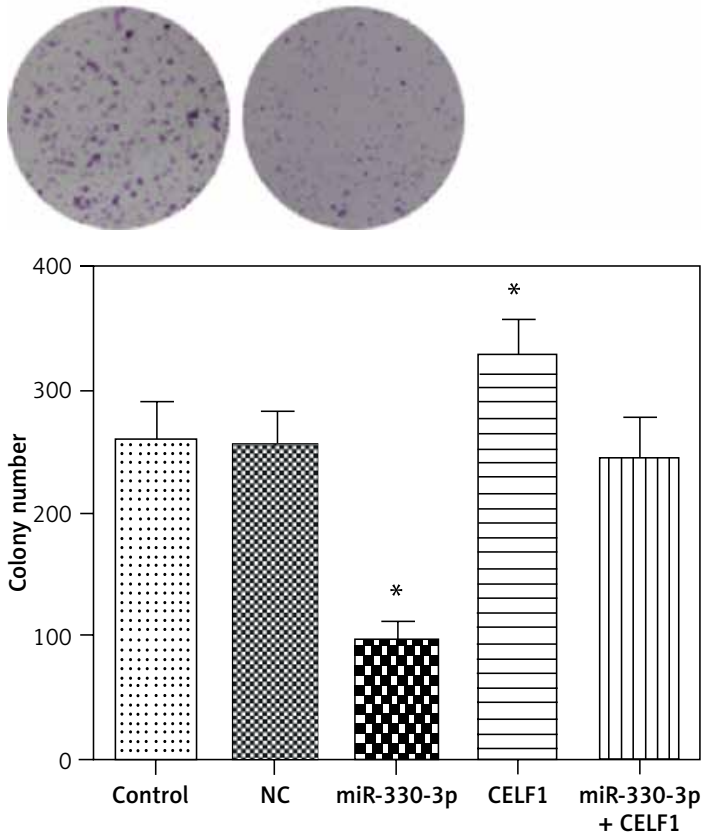
E
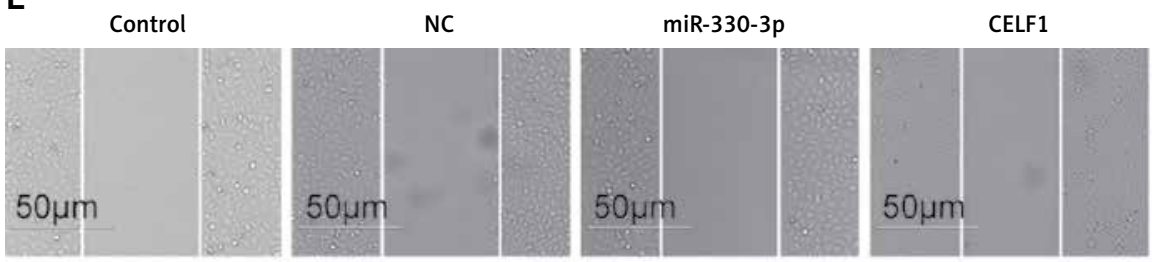

miR-330-3p + CELF1
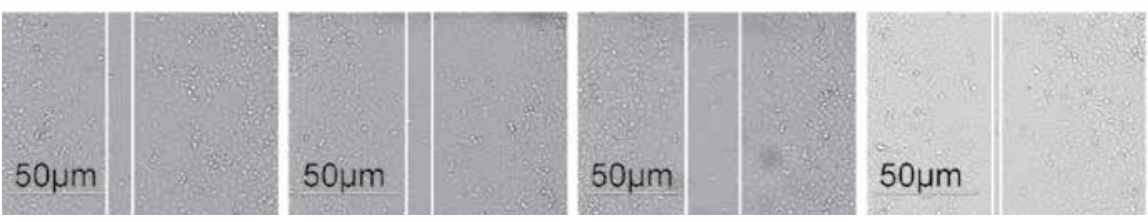

$50 \mu \mathrm{m}$

Figure 3. Cont. E - Wound healing assay showed that miR-330-3p overexpression significantly inhibited glioma cell migration, whereas CELF1 overexpression drastically enhanced the migration ability of glioma cell. MiR-330-3p could suppress glioma cell migration through downregulating CELF. ${ }^{*} P<0.05$, compared with the control group

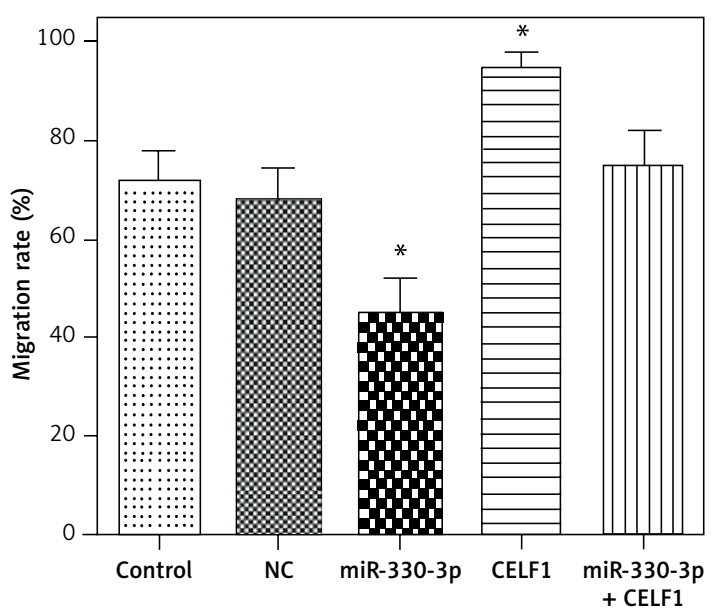

CELF1 group and the control group $\left({ }^{*} p>0.05\right)$. All the results above demonstrated that miR-330-3p might suppress glioma cell propagation and migration via down-regulating CELF1.

\section{Up-regulation of miR-330-3p inhibited tumor growth in vivo}

Xenograft tumor assay was used to test the effects of miR-330-3p on glioma in vivo. The tumor progression of glioma in vivo could be inhibited by up-regulation of miR-330-3p. Over-expression of miR-330-3p showed better performance in suppressing the volume of glioma tumor than the NC group, and simultaneously up-regulating miR330-3p minimized the tumor growth in nude mice $(p<0.01$, Figures 4 A-C).

\section{Discussion}

In the current study, we detected lower expression of miR-330-3p and higher expression of CELF1 in glioma tissues and cell lines. Furthermore, we validated the target relationship between miR-330-3p and CELF1 and demonstrated that miR-330-3p inhibited glioma cell reproduction and migration via down-regulation of CELF1 expression. These findings identified a new target for the therapeutic strategy of glioma and laid

a foundation for extensive study on the molecular mechanism of miR-330-3p in glioma cells.

There is considerable evidence suggesting that dysregulation of miRNAs could incur the aberrant expression of genes and tumorigenesis [10, 11, 27]. For instance, miR-637 was found significantly decreased in glioma tissues, which could suppress glioma cell growth and metastasis [28]. MiR-330 promoted the malignant behavior of glioblastoma stem cells and significantly activated both the ERK and PI3K/AKT signaling pathways [29]. Down-regulation of miR-145 expression suppressed metastasis of glioma stem cells by targeting ABCG2 in glioblastoma multiforme tumors [30]. However, miRNA can also act as a tumor facilitator in multiple cancer cells. Chen et al. found that miR106 could induce the proliferation of glioma cells through regulating the JNK/MAPK pathway [31]. Zhou et al. revealed that miRNA-155 acted as a facilitator of glioma cell proliferation via regulation of MXI1 [32]. Palumbo et al. studied different glioma cell lines and found the activity and the possibility to generate neurospheres [33]. Inspired by it, we used three glioma cells to find a proper subject to adapt the change of miR-330-3p and CELF1 and for the subsequent experiments. In our study, miR-330-3p was significantly lowly expressed in glioma specimens and cells. Further- 
A

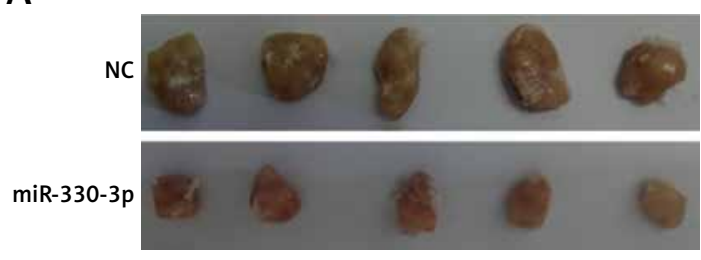

C

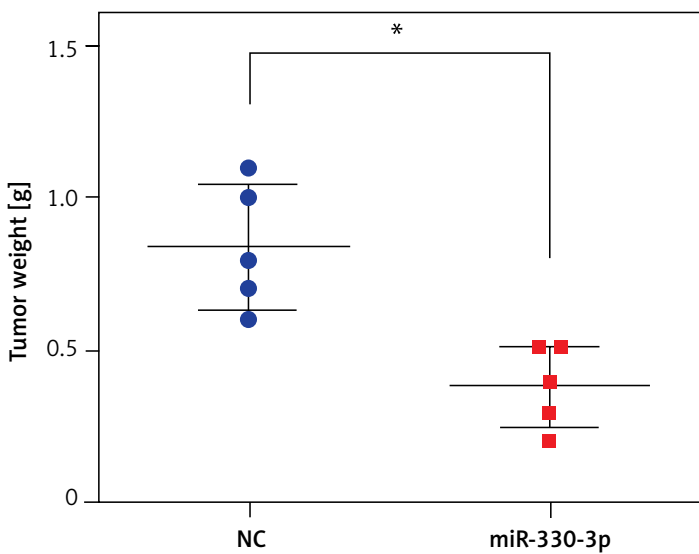

B

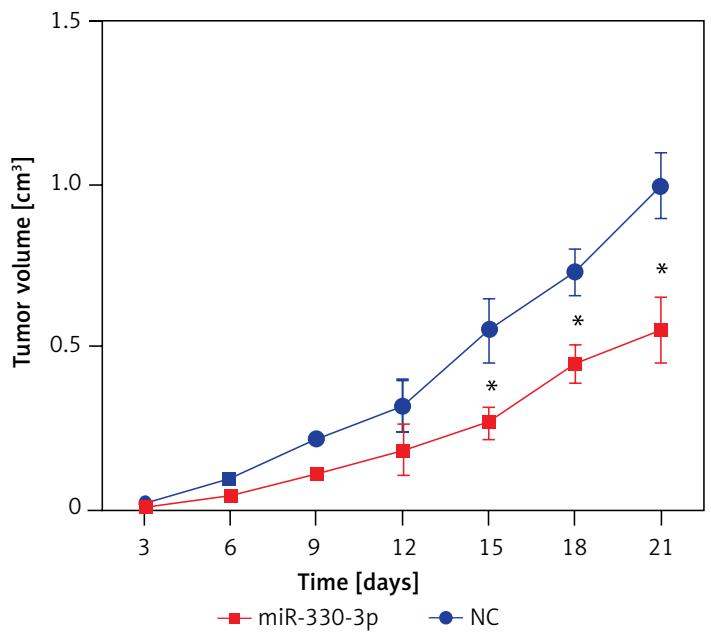

Figure 4. Up-regulation of miR-330-3p inhibited tumor growth in vivo. A - The xenograft model of nude mice transplanted with glioma cells showed that transfection of miR-330-3p mimics inhibited tumor growth in nude mice. B, C - The tumor volume and tumor weight in the miR-330-3p group were smaller than those in the NC group. ${ }^{*} P<0.05$, compared with the NC group

more, miR-330-3p overexpression could drastically repress the proliferation and migration of glioma cells. Combined with in vivo experiments, it was verified in our study that overexpression of miR-330-3p could suppress the tumor growth of glioma. Similar studies also indicated that miR330-3p can modulate gene expression in various cancers. For instance, miR-330-3p could promote metastasis in human breast cancer by targeting CCBE1 [20]. Nonetheless, there are few studies on the effects of miR-330-3p on glioma progression. In accordance with bioinformatic analysis and the dual-luciferase reporter assay, we further identified CELF1 as a functional target of miR-330-3p. Moreover, CELF1 expression in glioma tissues and cells could be repressed by ectopic expression of miR-330-3p, which was also consistent with a previous study [26].

Intriguingly, CELF1, as a broadly expressed member of the CUGBP ELAV-like family of RNA-binding proteins, regulates post-transcriptional gene expression by facilitating alternative splicing, translation as well as mRNA degradation, controlling cell growth, motility, and apoptosis [34-36]. House et al. reported that RNA-binding protein CELF1 could promote tumor growth and change gene expression in oral squamous cell carcinoma [37]. Rizzoli et al. demonstrated that CELF1 promoted glioma cell propagation via suppression of CDKN1B [38]. Talwar et al. found that overex- pression of CELF1 inhibited oral cancer cell apoptosis [39]. Gao et al. revealed that downregulation of CELF1 promoted apoptosis and suppressed cell proliferation of non-small cell lung cancer [40]. The previous study presented an 11-component genetic pathway, and showed that CELF1 protein functions as a central node controlling translational activation of genes driving EMT and ultimately tumor progression [41]. In our study, CELF1 was overexpressed in glioma tissues and cells. Zhong et al. found that the enhanced expression of syntenin and phosphorylated FAK may correlate with the increase of the malignancy of human gliomas [42]. In addition, the finding reported by Cui et al. identified miR-503 as both a novel regulator of CELF1 expression and a modulator of intestinal epithelial homoeostasis [43]. Furthermore, miR-214$3 p$ was found to act as a tumor suppressor and that its downregulation contributed to chemoresistance in esophageal cancer cells by targeting CELF1 [44]. We demonstrated that overexpressed miR-330-3p inhibited glioma cell migration by targeting CELF1. Furthermore, we also substantiated that the interplay between miR-330-3p and CELF1 played a crucial role in glioma development. MiR330-3p might inhibit glioma cell reproduction and migration by targeting CELF1.

Nevertheless, there are several limitations in the study. Firstly, the number of patients was not big enough. Secondly, miR-330-3p is not the only 
miRNA which regulates CELF1. As for this point, on line sequence target prediction sites are taken into consideration and bioinformatics analysis will be used in our further study. The tumorigenesis cell pathway miR-330-3p/CELF1 involved needs to be further studied.

In conclusion, miR-330-3p directly targeted CELF1 and might inhibit glioma cell reproduction and migration by down-regulating the expression of CELF1. The study might provide a new tumor marker for glioma diagnosis and prognosis.

\section{Conflict of interest}

The authors declare no conflict of interest.

\section{References}

1. Le Rhun E, Taillibert S, Chamberlain MC. Anaplastic glioma: current treatment and management. Expert Rev Neurother 2015; 15: 601-20.

2. Omuro A, DeAngelis LM. Glioblastoma and other malignant gliomas: a clinical review. JAMA 2013; 310: 1842-50.

3. Grimm SA, Chamberlain MC. Brainstem glioma: a review. Curr Neurol Neurosci Rep 2013; 13: 346.

4. Dietrich PY, Dutoit V, Walker PR. Immunotherapy for glioma: from illusion to realistic prospects? Am Soc Clin Oncol Educ Book 2014; 51-9.

5. Bergthold G, Bandopadhayay P, Bi WL, et al. Pediatric low-grade gliomas: how modern biology reshapes the clinical field. Biochim Biophys Acta 2014; 1845: 294-307.

6. Dhermain F. Radiotherapy of high-grade gliomas: current standards and new concepts, innovations in imaging and radiotherapy, and new therapeutic approaches. Chin J Cancer 2014; 33: 16-24.

7. Tutar L, Tutar E, Ozgur A, Tutar Y. Therapeutic targeting of microRNAs in cancer: future perspectives. Drug Dev Res 2015; 76: 382-8.

8. Acunzo M, Romano G, Wernicke D, Croce CM. MicroRNA and cancer - a brief overview. Adv Biol Regul 2015; 57: $1-9$

9. Pereira DM, Rodrigues PM, Borralho PM, Rodrigues CM. Delivering the promise of miRNA cancer therapeutics. Drug Discov Today 2013; 18: 282-9.

10. Rolle K. miRNA Multiplayers in glioma. From bench to bedside. Acta Biochim Pol 2015; 62: 353-65.

11. Wang BC, Ma J. Role of microRNAs in malignant glioma. Chin Med J 2015; 128: 1238-44.

12. Zhao B, Bian EB, Li J, Li J. New advances of microRNAs in glioma stem cells, with special emphasis on aberrant methylation of microRNAs. J Cell Physiol 2014; 229: 1141-7.

13. Zhang Y, Dutta A, Abounader R. The role of microRNAs in glioma initiation and progression. Front Biosci 2012; 17: 700-12.

14. Towler BP, Jones Cl, Newbury SF. Mechanisms of regulation of mature miRNAs. Biochem Soc Trans 2015; 43: 1208-14.

15. Tavares AL, Artinger KB, Clouthier DE. Regulating craniofacial development at the 3' end: microRNAs and their function in facial morphogenesis. Curr Top Dev Biol 2015; 115: 335-75.

16. Zhang J, Gong X, Tian K, et al. miR-25 promotes glioma cell proliferation by targeting CDKN1C. Biomed Pharmacother 2015; 71: 7-14.
17. Hu X, Chen D, Cui Y, Li Z, Huang J. Targeting microRNA-23a to inhibit glioma cell invasion via HOXD10. Sci Rep 2013; 3: 3423.

18. Wan X, Cheng Q, Peng R, et al. ROCK1, a novel target of miR-145, promotes glioma cell invasion. Mol Med Rep 2014; 9: 1877-82.

19. Shi ZM, Wang XF, Qian X, et al. MiRNA-181b suppresses IGF-1R and functions as a tumor suppressor gene in gliomas. RNA 2013; 19: 552-60.

20. Mesci A, Huang X, Taeb S, et al. Targeting of CCBE1 by miR-330-3p in human breast cancer promotes metastasis. Br J Cancer 2017; 116: 1350-7.

21. Guan A, Wang H, Li X, et al. MiR-330-3p inhibits gastric cancer progression through targeting MSI1. Am J Transl Res 2016; 8: 4802-11.

22. Meng H, Wang K, Chen X, et al. MicroRNA-330-3p functions as an oncogene in human esophageal cancer by targeting programmed cell death 4. Am J Cancer Res 2015; 5: 1062-75.

23. Rattenbacher B, Beisang D, Wiesner DL, et al. Erratum for Rattenbacher et al., Analysis of CUGBP1 targets identifies GU-repeat sequences that mediate rapid mRNA decay. Mol Cell Biol 2016; 36: 660.

24. Bohjanen PR, Moua ML, Guo L, Taye A, Vlasova-St Louis IA. Altered CELF1 binding to target transcripts in malignant T cells. RNA 2015; 21: 1757-69.

25. Zhou Y, Ma H, Fang J, Lian M, Feng L, Wang R. Knockdown of CUG-binding protein 1 induces apoptosis of human laryngeal cancer cells. Cell Biol Int 2014; 38: 1408-14.

26. Xia L, Sun C, Li Q, et al. CELF1 is up-regulated in glioma and promotes glioma cell proliferation by suppression of CDKN1B. Int J Biol Sci 2015; 11: 1314-24.

27. Tumilson CA, Lea RW, Alder JE, Shaw L. Circulating microRNA biomarkers for glioma and predicting response to therapy. Mol Neurobiol 2014; 50: 545-58.

28. Que T, Song Y, Liu Z, et al. Decreased miRNA-637 is an unfavorable prognosis marker and promotes glioma cell growth, migration and invasion via direct targeting Akt1. Oncogene 2015; 34: 4952-63.

29. Yao Y, Xue Y, Ma J, et al. MiR-330-mediated regulation of SH3GL2 expression enhances malignant behaviors of glioblastoma stem cells by activating ERK and PI3K/AKT signaling pathways. PLoS One 2014; 9: e95060.

30. Shi L, Wang Z, Sun G, Wan Y, Guo J, Fu X. miR-145 inhibits migration and invasion of glioma stem cells by targeting ABCG2. Neuromolecular Med 2014; 16: 517-28.

31. Chen XH, Ling XM, Shi S. microRNA-106a induces the proliferation and apoptosis of glioma cells through regulating JNK/MAPK pathway. Eur Rev Med Pharmacol Sci 2015; 19: 3412-7.

32. Zhou J, Wang W, Gao Z, et al. MicroRNA-155 promotes glioma cell proliferation via the regulation of MXI1. PLoS One 2013; 8: e83055.

33. Palumbo P, Miconi G, Cinque B, et al. NOS2 expression in glioma cell lines and glioma primary cell cultures: correlation with neurosphere generation and SOX-2 expression. Oncotarget 2017; 8: 25582-98.

34. Beisang D, Reilly C, Bohjanen PR. Alternative polyadenylation regulates CELF1/CUGBP1 target transcripts following T cell activation. Gene 2014; 550: 93-100.

35. Rattenbacher B, Beisang D, Wiesner DL, et al. Analysis of CUGBP1 targets identifies GU-repeat sequences that mediate rapid mRNA decay. Mol Cell Biol 2010; 30: 3970-80.

36. Kress C, Gautier-Courteille C, Osborne HB, Babinet C, Paillard L. Inactivation of CUG-BP1/CELF1 causes growth, viability, and spermatogenesis defects in mice. Mol Cell Biol 2007; 27: 1146-57. 
37. House RP, Talwar S, Hazard ES, Hill EG, Palanisamy V. RNA-binding protein CELF1 promotes tumor growth and alters gene expression in oral squamous cell carcinoma. Oncotarget 2015; 6: 43620-34.

38. Rizzoli U, Mangoni L, Carella AM, et al. Drug-mediated marrow purging: mafosfamide in adult acute leukemia in remission. The experience of the Italian study group. Bone Marrow Transplant 1989; 4 Suppl 1: 190-4.

39. Talwar S, Balasubramanian S, Sundaramurthy S, et al. Overexpression of RNA-binding protein CELF1 prevents apoptosis and destabilizes pro-apoptotic mRNAs in oral cancer cells. RNA Biol 2013; 10: 277-86.

40. Gao C, Yu Z, Liu S, Xin H, Li X. Overexpression of CUGBP1 is associated with the progression of non-small cell lung cancer. Tumour Biol 2015; 36: 4583-9.

41. Chaudhury A, Cheema S, Fachini JM, et al. CELF1 is a central node in post-transcriptional regulatory programmes underlying EMT. Nat Commun 2016; 7: 13362.

42. Zhong D, Ran J, Zhang X, et al. Syntenin is expressed in human gliomas and may correlate with tumor migration. Arch Med Sci 2015; 11: 1303-7.

43. Cui YH, Xiao L, Rao JN, et al. miR-503 represses CUG-binding protein 1 translation by recruiting CUGBP1 mRNA to processing bodies. Mol Biol Cell 2012; 23: 151-62.

44. Phatak P, Byrnes KA, Mansour D, et al. Overexpression of miR-214-3p in esophageal squamous cancer cells enhances sensitivity to cisplatin by targeting survivin directly and indirectly through CUG-BP1. Oncogene 2016; 35: 2087-97. 